\title{
Symmetry energy, neutron star crust and neutron skin thickness ${ }^{\star}$
}

\author{
Isaac Vidaña ${ }^{1}$. Constança Providência ${ }^{1}$. \\ Artur Polls ${ }^{2}$. Arnau Rios ${ }^{3}$
}

Received: date / Accepted: date

\begin{abstract}
We analyze the correlations of the slope and curvature parameters of the symmetry energy with the neutron skin thickness of neutron-rich isotopes, and the crust-core transition density in neutron stars. The results are obtained within the microscopic Brueckner-Hartree-Fock approach, and are compared with those obtained with several Skyrme and relativistic mean field models.
\end{abstract}

Keywords Symmetry energy · Neutron star crust · Neutron skin thickness

PACS 21.65.Cd $\cdot 21.65 . \mathrm{Ef} \cdot 21.65 . \mathrm{Mn}$

\section{Introduction}

Isospin asymmetric nuclear matter is present in nuclei, especially in those far away from the stability line, and in astrophysical systems, particularly in neutron stars. Therefore, a well-grounded understanding of the properties of isospin-rich nuclear matter is a necessary ingredient for the advancement of both nuclear physics and astrophysics. However, some of these properties are not well constrained yet. In particular, the density dependence of the symmetry energy is still an important source of uncertainties [1]. In this work, we analyze the correlations of the slope, $L=3 \rho_{0} \partial E_{\text {sym }} /\left.\partial \rho\right|_{\rho_{0}}$, and curvature, $K_{\text {sym }}=9 \rho_{0}^{2} \partial^{2} E_{\text {sym }} /\left.\partial \rho^{2}\right|_{\rho_{0}}$, parameters of the symmetry energy with the neutron skin thickness of neutron-rich isotopes, and the crust-core transition density in neutron star. The results are obtained within the microscopic Brueckner-HartreeFock (BHF) approach, and are compared with those obtained with several Skyrme and relativistic mean field (RMF) models.

* Presented at the 21st European Conference on Few-Body Problems in Physics, Salamanca, Spain, 30 August - 3 September 2010

${ }^{1}$ CFC, Department of Physics, University of Coimbra, PT-3004-516 Coimbra, Portugal ${ }^{2}$ Departament d'ECM, Universitat de Barcelona, Avda. Diagonal 647, E-08028 Barcelona, Spain

${ }^{3}$ Faculty of Engineering and Physical Sciences, Department of Physics, University of Surrey, Guildford, Surrey GU2 7XH, United Kingdom 

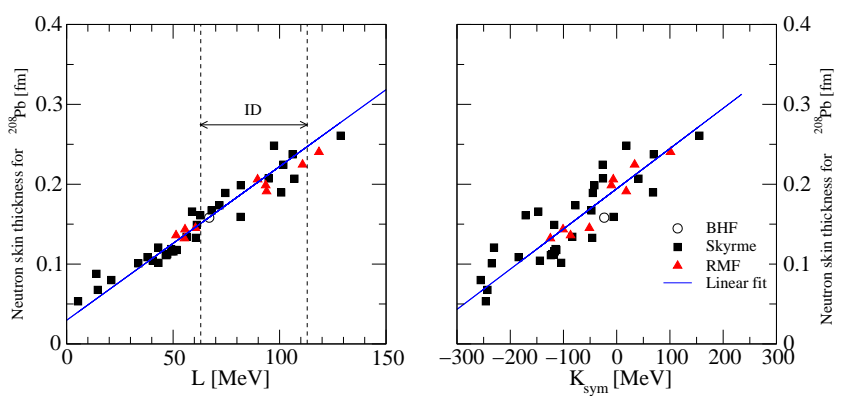

Fig. 1 Neutron skin thickness for ${ }^{208} \mathrm{~Pb}$ versus $L$ (left panel) and $K_{\text {sym }}$ (right panel). The vertical dashed lines on the left panel denote the constraints on $L$ from isospin diffusion (ID).

\section{The BHF approach of asymmetric nuclear matter}

Assuming charge symmetry of nuclear forces, the energy per particle of asymmetric nuclear matter can be written in good approximation in terms of the isospin asymmetry parameter, $\beta=\left(\rho_{n}-\rho_{p}\right) / \rho$, as $E / A(\rho, \beta) \sim E_{S N M}(\rho)+E_{\text {sym }}(\rho) \beta^{2}$, being $E_{S N M}(\rho)$ the energy per particle of symmetric nuclear matter, and $E_{\text {sym }}(\rho)=E / A(\rho, \beta=$ 1) $-E_{S N M}(\rho)$ the so-called symmetry energy.

The BHF approach of asymmetric nuclear matter starts with the construction of all the $G$ matrices describing the effective interaction between two nucleons in the presence of a surrounding medium. They are obtained by solving the Bethe-Goldstone equation

$$
G_{\tau_{1} \tau_{2} ; \tau_{3} \tau_{4}}(\omega)=V_{\tau_{1} \tau_{2} ; \tau_{3} \tau_{4}}+\sum_{i j} V_{\tau_{1} \tau_{2} ; \tau_{i} \tau_{j}} \frac{Q_{\tau_{i} \tau_{j}}}{\omega-\epsilon_{i}-\epsilon_{j}+i \eta} G_{\tau_{i} \tau_{j} ; \tau_{3} \tau_{4}}(\omega)
$$

where $\tau=n, p$ indicates the isospin projection of the two nucleons in the initial, intermediate and final states, $V$ denotes the bare NN interaction, $Q_{\tau_{i} \tau_{j}}$ the Pauli operator, and $\omega$ corresponds to the sum of non-relativistic energies of the interacting nucleons. The single-particle energy $\epsilon_{\tau}$ of a nucleon with momentum $\mathbf{k}$ is given by

$$
\epsilon_{\tau}(\mathbf{k})=\frac{\hbar^{2} k^{2}}{2 m_{\tau}}+\operatorname{Re}\left[U_{\tau}(\mathbf{k})\right]
$$

where $U_{\tau}(\mathbf{k})$ represents the mean field "felt" by a nucleon due to its interaction with the other nucleons of the medium. In the BHF approximation, $U(\mathbf{k})$ is given by

$$
U_{\tau}(\mathbf{k})=\sum_{\tau^{\prime}} \sum_{\left|\mathbf{k}^{\prime}\right|<k_{F_{\tau^{\prime}}}}\left\langle\mathbf{k k}^{\prime}\left|G_{\tau \tau^{\prime} ; \tau \tau^{\prime}}\left(\omega=\epsilon_{\tau}(k)+\epsilon_{\tau^{\prime}}\left(k^{\prime}\right)\right)\right| \mathbf{k k}^{\prime}\right\rangle_{A} .
$$

Once a self-consistent solution of Eqs. (1) and (3) is achieved, the energy per particle of asymmetric matter (and consequently the symmetry energy) can be calculated as

$$
\frac{E}{A}(\rho, \beta)=\frac{1}{A} \sum_{\tau} \sum_{|\mathbf{k}|<k_{F_{\tau}}}\left(\frac{\hbar^{2} k^{2}}{2 m_{\tau}}+\frac{1}{2} \operatorname{Re}\left[U_{\tau}(\mathbf{k})\right]\right) .
$$

For further reading, and details on the Skyrme forces and the relativistic models considered in this work, the reader is referred to Ref. [2]. 

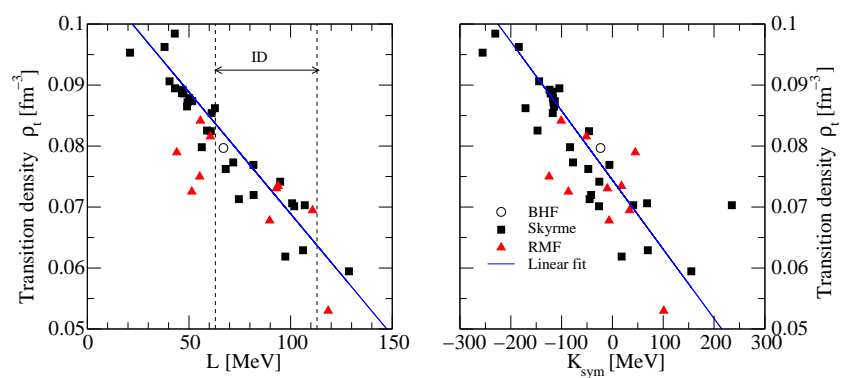

Fig. 2 Transition density from non-uniform to uniform $\beta$-stable matter as a function of $L$ (left panel) and $K_{s y m}$ (right panel). The vertical dashed lines on the left panel denote the constraints on $L$ from isospin diffusion (ID).

\section{Results}

It has been shown by Typel and Brown [3] that the neutron skin thickness, $\delta R=$ $\sqrt{\left\langle r_{n}^{2}\right\rangle}-\sqrt{\left\langle r_{p}^{2}\right\rangle}$ is very sensitive to the density dependence of the nuclear symmetry energy. In Fig. 1 we show the correlation between $\delta R$ for ${ }^{208} \mathrm{~Pb}$ with the parameters $L$ (left panel) and $K_{\text {sym }}$ (right panel). It can be seen that both the Skyrme forces and the relativistic models predict values of $\delta R$ that exhibit a tight linear correlation with $L$. Note that the microscopic Brueckner calculation is in excellent agreement with this correlation. The linear increase of $\delta R$ with $L$ is not surprising since the thickness of the neutron skin in heavy nuclei is determined by the pressure difference between neutrons and protons, which is proportional to the parameter $L, P\left(\rho_{0}, \beta\right) \sim L \rho_{0} \beta^{2} / 3$ [4].

Another sensitive quantity to the symmetry energy is the transition density $\rho_{t}$ from non-uniform to uniform $\beta$-stable matter. We display in Fig. $2 \rho_{t}$ as a function of the parameters $L$ and $K_{\text {sym }}$ for the BHF calculation together with the predictions of the several Skyrme forces and relativistic models. Using the experimental constraint on $L$ from isospin diffusion, we estimate the transition density to be between $0.063 \mathrm{fm}^{-3}$ and $0.083 \mathrm{fm}^{-3}$, in reasonable agreement with the value of $\rho_{t} \approx 0.08 \mathrm{fm}^{-3}$ often used in the literature.

\section{Summary}

We have studied the correlations of the slope and curvature parameters of the symmetry energy with the neutron skin thickness of neutron-rich isotopes, and the crust-core transition density in neutron stars. The results have been obtained within the microscopic Brueckner-Hartree-Fock approach, and have been compared with those obtained with several Skyrme and relativistic mean field models. A very good agreement between the different models has been found.

\section{References}

1. B. A. Li, L. W. Chen and C. M. Ko, Phys. Rep. 464, 113 (2008).

2. I. Vidaña, C. Providência, A. Polls and A. Rios, Phys. Rev. C 80, 045806 (2009).

3. S. Typel and B. A. Brown, Phys. Rev. C 64, 027302 (2001).

4. P. Danielewicz and J. Lee, Nucl. Phys. A 818, 36 (2009). 